\title{
Implementing the IMU professionalism education framework in the MBBS programme
}

\author{
Thuraisingham, C., Krishnappa, P., Sui, C.Y., Ismail, N.B.H.M. \\ International Medical University, Malaysia
}

\section{Background:}

Professionalism is a key component of medical training. Standards of professional behaviour must be internalized by students during their undergraduate training in readiness for the workplace. In the past, professionalism was introduced informally through "the hidden curriculum". Today, it is advocated that medical graduates must be formally trained in professionalism. IMU's goal is to make professionalism an explicit component of the MBBS curriculum so that all graduates attain a satisfactory level.

\section{Methodology:}

The IMU Professionalism Framework with its three overarching domains, namely, individual proficiency, client-centeredness, and work-centeredness, was amalgamated with the draft of the National Competency Framework (dNCF) the gold standard for practicing doctors in Malaysia. The amalgamation required much planning which was scientifically and meticulously carried out by a Working Group comprising members of IMU's Medical and Clinical Sciences. Essential attributes from each domain were identified. These were mapped to the dNCF list of 9 attributes, and to the School of Medicine's teaching and learning activities from Semester 1 to 10 , indicating the level of competency expected at each semester. Methods of evaluation were also outlined. The final matrix reflected students' level of professionalism as they progressed from one semester to the next in the Framework. In the dNCF document, upon graduation students are expected to be at Level 4, and this was also reflected in the IMU Framework. The matrix will be able to demonstrate when and where every attribute would be taught and assessed explicitly, showing how teaching and evaluation of professionalism is carried out within the IMU MBBS curriculum.

\section{Conclusion:}

Students will be monitored through a 'tracking system'. A reward system, with certification, will be incorporated to celebrate achievements and attainment of professional behaviour at the end of the Medical and Clinical Sciences. This IMU Professionalism Education Framework will be implemented in August 2021.

Keywords: Professionalism, Reward 